\section{Military use of vaccine criticized}

Washington

NEARLY four years after smallpox was universally eradicated, the US military is still administering the vaccine as a "defensive and deterrent measure" against the use of smallpox virus as a biological weapon. This policy has put the US Army indirectly at odds with the World Health Organization and the US Centers for Disease Control (CDC), both of which have been working to eliminate all use of the vaccine. 'We've had several deaths and significant morbidity from its use for non-indicated reasons', says Dr Stanley Foster, assistant director of CDC's international health programme office. The main civilian users have been physicians who either did not know that smallpox had been wiped out or who believed that smallpox vaccine was effective against genital herpes. CDC last spring persuaded Wyeth Laboratories, the only remaining manufacturer of the vaccine in the United States, to take the product off the commercial market. Wyeth had at last count been selling some 50,000 doses per year.

Wyeth is, however, continuing to supply the military. All active duty personnel and reserves are vaccinated on a five-year schedule - which amounts to several hundred thousand doses per year. The military has also requested Wyeth to supply a stockpile of the vaccine sufficient to dose a classified number of personnel in the case of a general call-up.

Adverse reactions to the vaccine, which contains live vaccinia virus, are rare, but not insignificant. According to CDC, one death can be expected for every million eczema ( 38 per million). from the military. persons vaccinated. Generally less severe reactions (although sometimes fatal themselves) occur more frequently, mostly in certain susceptible groups - immunocompromised subjects (about 7 reactions per million vaccinations) and those with

Dr James Kirkpatrick, an army lieutenant colonel who advises the Army Surgeon General's office on the smallpox vaccination programme, says that by exempting personnel with eczema (or who have a family member with eczema), the military has kept the rate of adverse reactions quite low. All vaccinations (including measles, rubella, tetanus, smallpox and a half dozen others) resulted in 60 hospital admissions a year, he said, with an average hospital stay of two days. Although no numbers are available for the rate of adverse reactions specifically from smallpox vaccinations, CDC says it receives about one request per month for vaccinia immune globin, which is used to treat reactions to the vaccine. All such requests since last spring have been

The military's reason for vaccinating against smallpox appears to be intelligence information that the Warsaw Pact nations and the People's Republic of China vaccinate their troops. "Nobody can rule out the possibility"' that smallpox would be used as a weapon, Kirkpatrick says, and it "would not be prudent" to stop vaccinating. According to $\mathrm{CDC}$, only the United States and the Soviet Union continue to maintain stocks of the smallpox virus. South Africa recently and with much fanfare announced the destruc-

\section{US dioxin question is tackled}

\section{Washington}

A MAJOR new programme to deal with dioxin has been unveiled by the Environmental Protection Agency (EPA), fulfilling a promise made by William Ruckelshaus when he became its administrator last spring. The programme will include investigations at hundreds of chemical plants and waste disposal sites at a cost of up to $\$ 250$ million over four years. Alvin Alm, EPA's deputy administrator, says the agency regards dioxin as one of the most perplexing and potentially dangerous chemicals ever to pollute the environment. The programme would concentrate on what was considered the most toxic of the 75 dioxin isomers $-2,3,7,8-T C D D$, which is known to cause chloracne in humans. In laboratory animals, it has been known to cause cancer, reproductive failure, reduced effectiveness of the immune system and significant changes in enzyme systems. EPA's Cancer Assessment Group says it should be regarded as both an initiator and a promoter of cancer.
Richter to head SLAC

\section{Washington}

BURTON Richter has been named to succeed Wolfgang Panofsky as director of the Stanford Linear Accelerator Center (SLAC). Richter, who won the Nobel Prize for Physics in 1976 (with C.C. Ting) for the discovery of the psi particle, will take up the post next September. Richter is the designer and prime mover of SLAC's linear collider, on which construction began two months ago. The $\$ 113$ million machine consists of a one-and-a-half mile loop attached to the main linear accelerator; electrons and positrons accelerated in the linear stretch will collide in the loop at centre-of-mass energies of up to $100 \mathrm{GeV}$. The linear collider, like the much larger LEP machine under construction at the European Organisation for Nuclear Research (CERN) at Geneva, will find its greatest application in detailed studies of the $Z^{0}$ particle. The electron-positron collisions are much cleaner and easier to interpret than the proton-antiproton collision used at CERN last year to detect the heavy bosons ( $Z^{0}$ and $\left.W^{+} / W_{-}\right)$. Unlike LEP, however, the linear collider will be breaking new ground in accelerator design - a design that Richter says will yield considerable cost savings over LEP's traditional ring structure.

Panofsky, who has directed SLAC since 1961 , decided to follow his own policy of retirement at the age of 65 for the laboratory's administrators but will act as \& technical adviser. Stephen Budiansky

tion of its stocks.

Although CDC has been careful not to criticize the military programme directly, CDC officials are known to be uneasy. And a number of smallpox researchers have questioned whether the supposed threat of smallpox as a biological weapon outweighs the known risks of vaccination. Smallpox has an 8-10 day incubation period and is not very infectious. Epidemiologic evidence shows that $30-40$ per cent of susceptible members of a household will catch the disease from an infected member, compared with 80-90 per cent for measles, for example. They also point out that smallpox virus would live only a matter of hours in an aerosol form, the presumed method of delivery as a weapon.

The use of smallpox vaccine by civilian physicians as an unorthodox treatment for genital herpes has been a bizarre footnote to the story. Controlled studies have demonstrated no benefit in preventing or healing herpes lesions; CDC has nonetheless received many calls from herpes patients protesting against CDC's role in removing the vaccine from the market and claiming that the vaccine was the only thing that had helped them. CDC officials say that some physicians had also apparently been using the vaccine as a general "tonic", supposedly to boost a patient's immune defences.

Stephen Budiansky 